\title{
Selected aspects of neutron decay
}

\author{
Stephan Paul \\ Physik Department E18 \\ Technische Universität München \\ James Frank Strasse, D-85748 Garching
}

\begin{abstract}
Precision measurements of neutron decay offer complementary access to particle physics at small distance scales or high energies. In particular they allow tests of the $\mathrm{V}$-A structure of the weak interaction. Among many experimental activities which are ongoing around the world we present two new experiments which are planned or studied for the near future. While the neutron lifetime still bears significant experimental uncertainties and thus has to be studied with greatest precision the two-body decay $(n \rightarrow H \bar{\nu})$ of the neutron has not yet been observed. Despite its small branching fraction it offers many possibilities in the framework of particle physics. Both cases are addressed in this contribution.
\end{abstract}

\section{Introduction}

The process underlying neutron decay is the semileptonic transition $d \rightarrow u e^{-} \bar{\nu}$. The Hamiltonian for this process is written as

$$
H=\frac{G_{F}}{\sqrt{2}} V_{u d} \cdot \bar{e}\left(1-\gamma_{5}\right) \nu \cdot \bar{u}\left(1-\gamma_{5}\right) d \text { with } \frac{G_{F}}{\sqrt{2}}=\frac{g^{2}}{8 M_{W}^{2}} \text { and } g \cdot \sin \vartheta_{w}=e
$$

and describes the usual V-A coupling known for weak interaction. Here, $V_{u d}$ describes the quark mixing (see CKM matrix), $G_{F}$ is the Fermi coupling constant, $M_{W}$ is the mass of the W-boson, $\sin \vartheta_{W}$ the Weinberg angle and e the electric charge. The two terms describe the lepton and quark transition amplitudes. However, as there is no free quark decay the process to be considered is more complicated (see fig.1).

In the hadronic environment the coupling constants turn into formfactors, which however are evaluated at very small $q^{2} \rightarrow 0$. The quark part of the Hamiltonian thus has to be rewritten as

$$
\begin{gathered}
V_{\mu}+A_{\mu}= \\
V_{u d} \cdot \overline{\psi_{p}}\left(f_{1}\left(q^{2} \rightarrow 0\right) \gamma_{\mu}+f_{2}\left(q^{2} \rightarrow 0\right) \frac{\sigma_{\mu \nu}}{m_{p}} q^{\nu}+f_{3}\left(q^{2} \rightarrow 0\right) \frac{q^{\mu}}{m_{p}}\right) \psi_{n}+\overline{\psi_{p}}\left(f_{i} \rightarrow g_{i}\right) \psi_{n}
\end{gathered}
$$




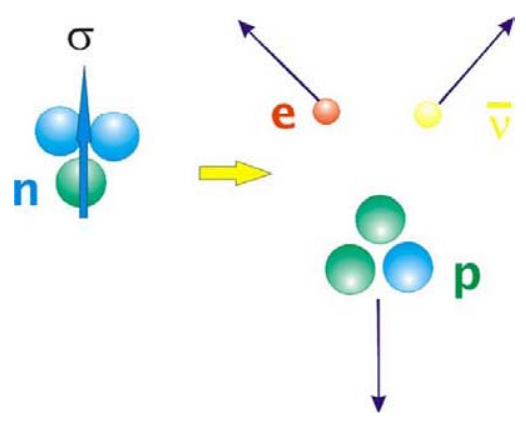

Figure 1: Sketch of the standard neutron decay

Using conservation of vector current and evaluating the expression at $q^{2} \rightarrow 0$ leaves only two terms

- $G_{V}=f_{1}\left(q^{2} \rightarrow 0\right) \cdot G_{F} \cdot V_{u d}=g_{V} \cdot G_{F} \cdot V_{u d}$

- $G_{A}=g_{1}\left(q^{2} \rightarrow 0\right) \cdot G_{F} \cdot V_{u d}=g_{A} \cdot G_{F} \cdot V_{u d}$

all other contributions vanish. For simplicity we define another quantity, called $\lambda$, with

$$
\lambda=\frac{G_{A}}{G_{V}}=\frac{g_{A}}{g_{V}}
$$

If we take $G_{F}$ from muon decay we are left with two independent 'free' parameters, $V_{u d}$ and $\lambda$. These parameters can be determined experimentally combining different measurements in neutron decay, decay asymmetries and the total decay rate using the neutron lifetime. The latter one relates to our free parameters via

$$
\tau \propto \frac{1}{G_{V}^{2} \cdot\left(1-3 \lambda^{2}\right)}
$$

Experimentally two different approaches have been taken to measure the neutron lifetime.

- Until the late eighties most experiments have used the in-beam method which requires the detection of $\mathrm{n}$-decay products from a well defined fiducial volume. Although being competitive on the statistical accuracy this method faces many systematic problems which has limited the final precision of the experiments.

- The most precise experiments have been performed using the method of stored neutrons. Ultra cold neutrons (UCN) can be confined in bottles by means of their interaction with the surface, the gravitational field and magnetic field gradients. Choosing proper materials neutrons are reflected from the surface with minimal losses which may occur either by absorption or by up-scattering (in which case 
they gain sufficient energy to leave the containing volume). The maximal allowed energies of such neutrons is around $250-300 \mathrm{neV}$. The neutron lifetime is derived from the number of neutrons extracted from this bottle after various storage times t. Varying the momentum spectrum of the neutrons one can estimate the loss rate in the bottle due to effects other than weak decay. These experiments have the virtue of high statistical accuracy (though still limited by the strength of the UCN sources available). The average lifetime extracted from many of such experiments is $885.7 \pm 0.8 s[1]$. Very recently, however, a new result has been published deviating from the present world average by about six standard deviations [2]. As the basic setup of this last experiment is almost identical to one of the previous measurements [3] it indicates that systematic effects, although claimed by all authors to be well under control, still are a major issue.

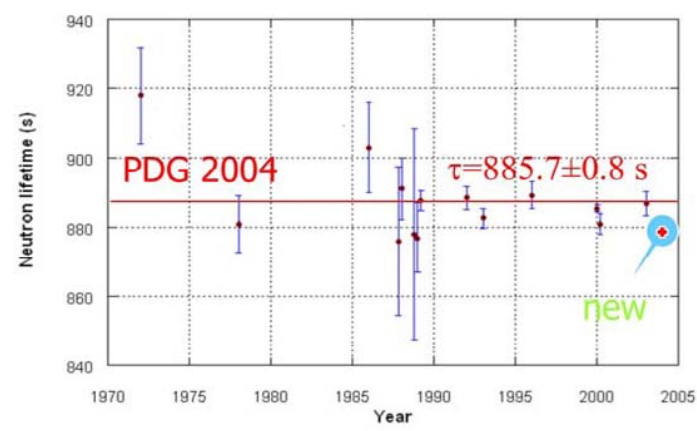

Figure 2: Experimental results for the neutron lifetime and the variation with time. Note the latest result which deviates from the world average by about 6 standard deviations.

We can now combine the results for the n-lifetime experiment as well as the neutron decay asymmetries for the electrons (not discussed here) to extract the quantities $V_{u d}$ and $\lambda$. This is shown in fig. 3, taken from [4]. $V_{u d}$ can also be determined by other methods, e.g. superallowed $0^{+} \rightarrow 0^{+}$nuclear beta decays, which offer superb statistical accuracy but suffer from systematic uncertainties connected to corrections for the nuclear medium. In addition, we can compare the direct measurements for $V_{u d}$ with the results from $V_{u b}$ and $V_{u s}$ in combination with the unitarity of the CKM matrix. Both values are also shown in fig 3. The present data set shows a clear disagreement of the different methods. This can have several reasons. It may hint to a non-unitarity of the CKM matrix or to the imcompleteness of the Hamiltonian. Thus, other contributions like right-handed currents or scalar and tensor interactions could play a role. On the other hand, experimental problems are not yet excluded as can be seen from the recent lifetime results which by itself would cure the present disagreement. Very recently a new analysis of neutral $\mathrm{K}$-decays $\left(\mathrm{K}_{l 3}\right)$ has given new values of $V_{u s}$, higher than the previous results which in 
turn lowers the expectations for $V_{u d}$ values extracted from the unitarity assumption [5]. But also there, systematic uncertainties, mainly related to theoretical calculations in the framework of chiral perturbation theory are at play. Taken at face value, the most recent results in kaon physics would by itself also cure the disagreement but would disfavor the new lifetime result.

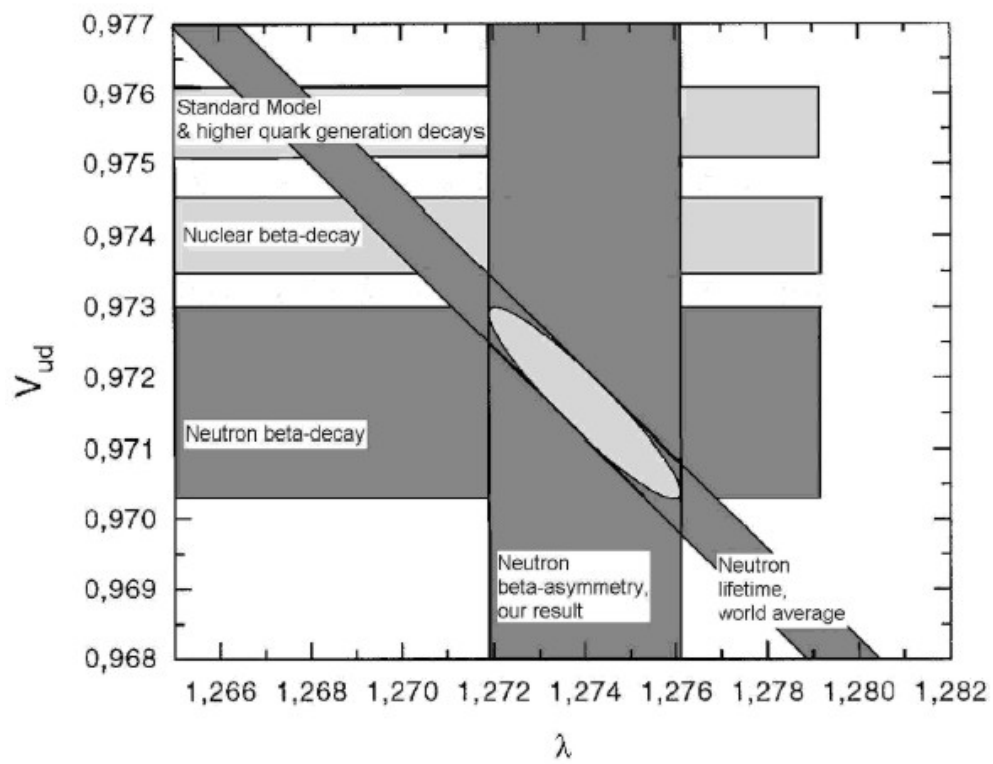

Figure 3: Experimental determination of the two standard parameters in neutron decay, $\mathrm{V}_{u d}$ and $\lambda[4]$.

It becomes clear from this discussion that new measurements are needed with emphasis on the understanding of systematic effects.

\section{A new neutron lifetime experiment}

A few years ago the group of the TUM has proposed a new experiment to measure the n-lifetime based on the use of a magnetic trap for ultra cold neutrons [6], [7]. This trap offers the unique possibility to simultaneously detect decay protons in real time as well as neutrons which had suffered spin flip during storage, the only foreseeable loss mechanism in a magnetic trap. The principle of this trap is shown in fig. 4. The trap consists of 19 super-conducting coils powered with alternating current direction thus forming a magnetic multipole system with inner diameter of $50 \mathrm{~cm}$. The forces in such a trap acting on the neutron are described by 


$$
\vec{F}=-\vec{\nabla}(\vec{\mu} \cdot \vec{B})
$$

where the magnetic moment of the neutron $\mu=-60.5 \mathrm{neV} / \mathrm{T}$ results in a repulsive force when $\vec{\mu}$ and $\vec{B}$ are parallel. Neutrons with this spin orientation are repelled from the walls which are at a maximal magnetic field of about $2 \mathrm{~T}$. In order to avoid spin flip processes in low field regions a cylindrical field is superimposed which is generated by a current rod in the center of the cylindrical trap. The current rod is shielded from the neutrons again by means of a multipole field generated by 18 current loops. The upper lid of the trap, which is at a height of about $1.2 \mathrm{~m}$, is formed by the gravitational force. Neutrons with energies up to $120 \mathrm{neV}$ can be stored in the trap.

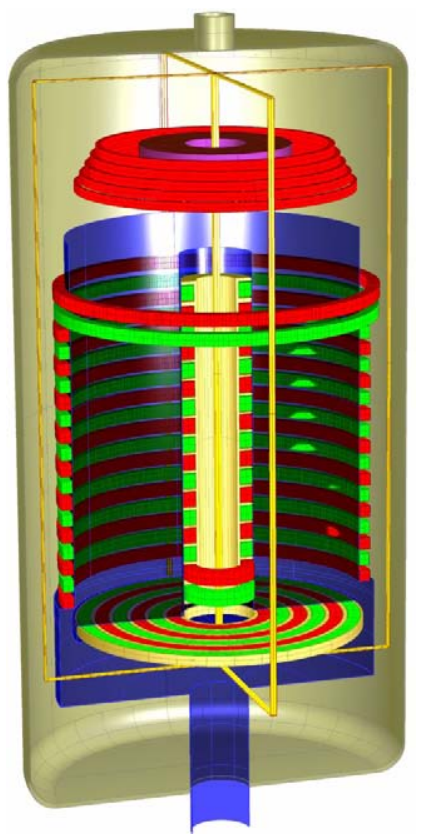

Figure 4: The proposed neutron lifetime experiment of the TUM. Neutrons can be stored inside a magnetic trap formed by two concentric cylindrical multipole fields. The trap can be filled (emptied) by a slit at the bottom which is open for all neutrons when the magnetic field is ramped down.

Neutrons are filled into the trap from a slit in the lower part of the setup which is connected to the UCN buffer volume (UCN source). It is situated between the outer torus and the bottom lid. The slit can be opened and closed magnetically by ramping the field structure within $100 \mathrm{~s}$.

In the first 150s of the filling neutrons are stored by means of a reflective layer mounted on the substrate which also serves a generator for an electric field. A thin cylinder 
surrounds the inner and outer coils separating them mechanically from the storage volume. On the side pointing away from the storage volume a conductive structure is mounted to form the field lines for the electrical field used to collect the decay protons. The inner side will be coated with a neutron reflective material. The initial phase of the storage cycle is also used for spectrum cleaning. Fig. 5 (left) shows the typical path of a neutron in the bottle when operated in storage mode. The path of decay protons is shown in fig. 5 (right). They are extracted towards the top by means of an electrical field of $20 \mathrm{kV}$ and a magnetic field with a component along the electrical field lines. After passing a focussing magnet (at the top) they are further accelerated onto a proton detector.
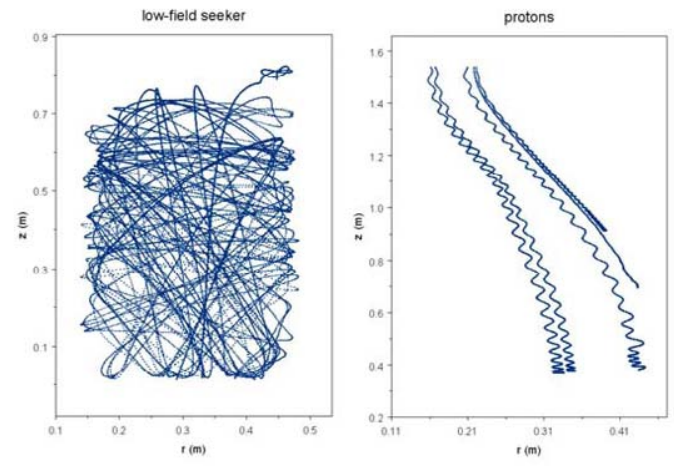

Figure 5: Left: Result of a ray tracing program for a neutron stored inside the magnetic trap. Right: Result of a ray tracing program for decay protons inside the magnetic trap.

The trap is foreseen to be installed at one of the newly planned high power UCN sources (preferentially at the FRMII). We expect to store about $10^{8}$ neutrons per measuring cycle in the volume of $700 \mathrm{l}$. Using the time spectrum of the decay protons a measuring accuracy of 1 second can be achieved per cycle (about $40 \mathrm{~min}$ ) [8]. Thus, the goal of $\delta t_{\text {stat }} \sim 0.1 \mathrm{~s}$ can be achieved within 3-4 days. Much effort has been devoted to allow enough flexibility to study systematics. Spin flip neutrons (if created at all) can be detected with an efficiency of about $60 \%$, stored neutrons with almost $100 \%$ and decay protons with $70 \%$. The momentum spectrum of the stored neutrons can be altered prior to each measuring cycle as well as the spring tension (storing B-field). We expect to operate this instruments in 2-3 years from now.

\section{The two-body decay of the neutron $\left(n \rightarrow H e^{-}\right)$}

Although neutron decay is almost uniquely passing as three body decay $\left(n \rightarrow p e^{-} \bar{\nu}\right)$ a small but important phase space exists for the charged particle to form a hydrogen atom 


\begin{tabular}{c|c|c|c|c|c|c|c|c|c} 
config. i & $\bar{\nu}$ & $\mathrm{n}$ & $\mathrm{p}$ & $e^{-}$ & transition & $W_{i}(\%)$ & $F$ & $m_{F}$ & $\left|m_{S} m_{I}\right\rangle$ \\
\hline 1 & $\leftarrow$ & $\leftarrow$ & $\leftarrow$ & $\rightarrow$ & $\mathrm{Fe}$ & $44.174 \pm .017$ & 0,1 & 0 & $|+-\rangle$ \\
2 & $\leftarrow$ & $\leftarrow$ & $\rightarrow$ & $\leftarrow$ & $\mathrm{GT}$ & $55.211 \pm .013$ & 0,1 & 0 & $|-+\rangle$ \\
3 & $\leftarrow$ & $\rightarrow$ & $\rightarrow$ & $\rightarrow$ & $\mathrm{Fe}$ & $.615 \pm .003$ & 1 & 1 & $|++\rangle$ \\
4 & $\rightarrow$ & $\leftarrow$ & $\leftarrow$ & $\leftarrow$ & $\mathrm{Fe}$ & 0. & 1 & -1 & $|--\rangle$ \\
2 & $\rightarrow$ & $\rightarrow$ & $\rightarrow$ & $\leftarrow$ & $\mathrm{Fe}$ & 0. & 0,1 & 0 & $|-+\rangle$ \\
1 & $\rightarrow$ & $\rightarrow$ & $\leftarrow$ & $\rightarrow$ & $\mathrm{GT}$ & 0. & 0,1 & 0 & $|+-\rangle$
\end{tabular}

Table 1: Spin projections $\mathrm{i}$ in the neutron bound $\beta$ decay. As a convention, the $\mathrm{H}$ moves to the right, the $\bar{\nu}$ to the left. $W_{i}$ are the populations according to pure V-A interaction, $F$ the total spin (with hyperfine interaction) and $m_{F}$ the $F$ projection, $\left|m_{S} m_{I}\right\rangle$ the Paschen-Back state, where $m_{S}$ and $m_{I}$ denote the $e^{-}$and $p$ spin quantum numbers.

in which case the kinematics of a two-body decay has to be considered. This two-body kinematics bears a number of very interesting features. If we consider massless neutrinos only, as well as left-handed currents (standard model) the population of the hyperfine states of the hydrogen atom is well predictable. In addition, the direction of flight of the hydrogen atoms defines the quantization axis for all angular momenta involved (when using unpolarized neutrons). As the weak interaction is very short ranged, all hydrogen atoms will be formed in a relative S-state $(\mathrm{nS})$ with the population of the various n-values to be governed again by phase space and kinematics. The hyperfine state of the hydrogen atom is directly related to the relative spin orientation of proton and electron and thus to their helicities. If the decay happens inside a small magnetic field, the helicities of all particles is conserved. Thus, an analysis of the different possible hyperfine states gives information about the validity of the assumptions made above.

Table 1 denotes the population of the different hyperfine states [10]. They are ordered in terms of helicities and will mix, if no magnetic field were present. As a convention, the $\mathrm{H}$ moves to the right, the $\bar{\nu}$ to the left. $F e$ and GT mean Fermi and Gamov-Teller transition, respectively. $W_{i}$ are the populations according to pure V-A interaction ([10][11]), $F$ the total spin (with hyperfine interaction) and $m_{F}$ the $F$ projection, $\left|m_{S} m_{I}\right\rangle$ the PaschenBack state, where $m_{S}$ and $m_{I}$ denote the $e^{-}$and $p$ spin quantum numbers (+ means $+1 / 2$, i. e., spin points to the left in the magnetic quantization field direction).

In order to see the sensitivity to an admixture of other interactions (like scalar or tensor) table two shows the modified population in case of such small admixtures [14].

The absence of a direct population of configuration 4 is striking. One can show, that this helicity configuration can only be populated if either the neutrino mass is finite or if a right handed admixture to the weak interaction exists. The latter can be formulated quantitatively by the following expressions. If we only assume the existence of a right handed $W_{R}$ with mass $M_{R}$ then the corresponding coupling constant $g_{R}$ scales with $M_{R}^{2}$, the relative probability with $M_{L}^{2} / M_{R}^{2}$ multiplied with a factor $(1+\lambda)^{2} /\left(2\left(1+3 \lambda^{2}\right)\right) \ll 1$ from spin gymnastics [12].

We may also consider left right symmetric models [13] where two mass eigenstates 


\begin{tabular}{c|c|c|c} 
config. i & $g_{S}=0, g_{T}=0$ & $g_{S}=0.1, g_{T}=0$ & $g_{S}=0, g_{T}=0.02$ \\
\hline 1 & 44.174 & 46.479 & 43.440 \\
2 & 55.211 & 53.288 & 55.789 \\
3 & .615 & .233 & .771 \\
4 & 0. & 0. & 0.
\end{tabular}

Table 2: $W_{i}(\%)$ for various $g_{S}$ and $g_{T}$.

$W_{1}$ and $W_{2}$ mix to form $W_{L}$ and $W_{R}$ with a mixing angle $\xi$ and mass ratio $\eta=\left(W_{1} / W_{2}\right)^{2}$. Then the contribution to configuration 4 will be

$$
W_{4}=2(\eta-\xi+\lambda(\eta+\xi))^{2}
$$

At any rate, $W_{4}$ is expected to be small, $W_{4}<10^{-7}$. Thus a very sensitive zero measurement is required where we have to also consider feeding of this state by atomic cascading from higher lying S-states, though populated with small probability.

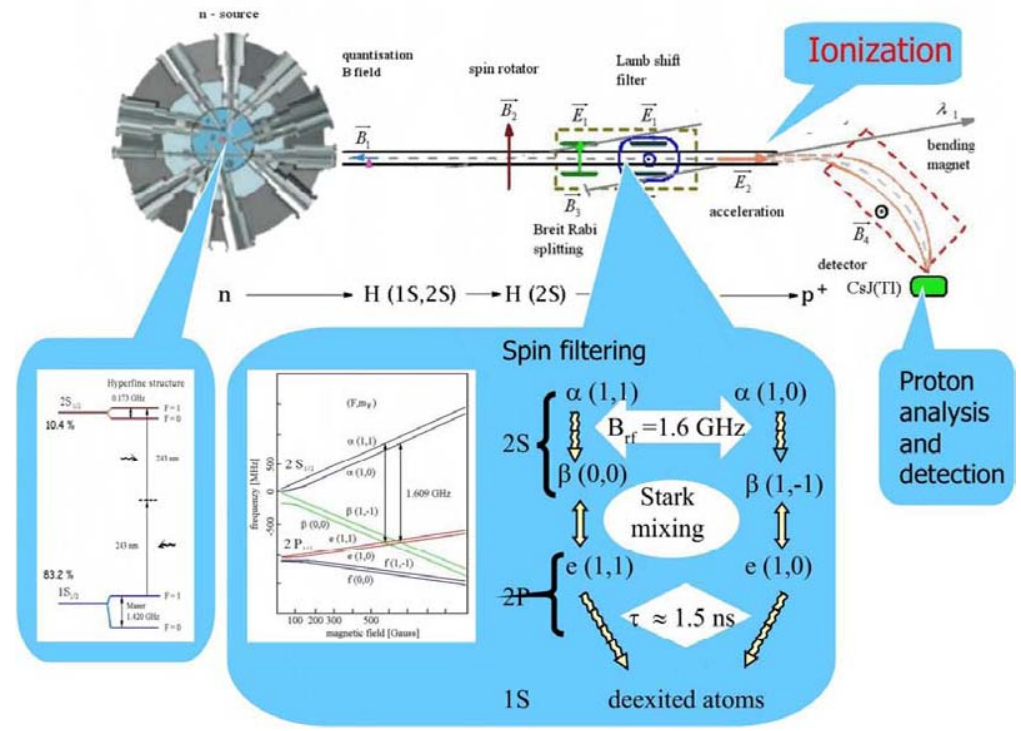

Figure 6: Setup

The experimental method [9] requires a strong neutron source, a large decay volume and background free analysis of the emerging hydrogen atoms. A possible setup is sketched 
in fig. 6 which depicts a typical research reactor with a tangential but through-going beam tube acting as decay volume. Hydrogen atoms are thus extracted without direct sight to the reactor core strongly reducing background from emerging neutrons. After a few meters flight path (within a small axial magnetic field) the hydrogen atoms pass through a lamb-shift polarimeter, where one can select one particular hyperfine state. The surviving hydrogen atoms are detected by resonance ionization and subsequent detection of the proton. The large Doppler-shift owing to the kinetic energy of the hydrogen atoms of $323 \mathrm{eV}$ is used to discriminate against rest gas atoms.

Owing to presently existing laser powers in the UV range, only hydrogen atoms produced in the $\mathrm{n}=2$ meta-stable state can be used, constituting about $10 \%$ of all 2 -body decays. The splitting of these states in the analyzing field of the lamb shift filter is shown in figure 6 (inlet) Using a high-power laser and a cavity we can induce transitions between $\alpha$ and $\beta$-states by means of an rf field of $1.609 \mathrm{GHz}$. Using an electric field of about 4.3 $\mathrm{V} / \mathrm{cm}$ for Stark-mixing we can control the quenching of the $\beta$-states to the short living $2 \mathrm{P}$ states (e-states) and thus depopulate selectively particular $\alpha$ and $\beta$-states [16]. Reversing the sign of the magnetic field in the decay region, the association of the hyperfine states to $\alpha$ and $\beta$-states can be reversed allowing access to all possible helicity configurations.

As there is no external trigger, the passage of a fast moving hydrogen atom is detected via its two step ionization. In a first step a Doppler-detuned laser induces the transition $2 S \rightarrow 3 P$ and a subsequent broad band light source ionizes the atom. The resulting proton will be accelerated in an electric field and the momentum analyzed in a spectrometer magnet equipped with a proton detector.

We can estimate measuring accuracies assuming a typical neutron flux as available at the FRMII in Munich of $2 \cdot 10^{14} \mathrm{~cm}^{-2} \mathrm{~s}^{-1}$ [18], [19]. This results in rate of detectable hydrogen atoms of $3 \mathrm{~s}^{-1}$ of which $10 \%$ are usable (2S). Assuming high efficiencies $\varepsilon$ (thus high laser power) we can improve the current limits of $g_{S}$ and $g_{T}$ by a by a factor $2.5 /$ day $\times \varepsilon$. Present limits are $g_{S}<6 \cdot 10^{-2}[20]$ and $g_{T}<0.125$ [21]. For the forbidden decay, reachable limits are of the order of $10^{-6} / \mathrm{yr}$.

\section{References}

[1] S. Eidelman et al. [Particle Data Group], Phys. Lett. B 592, 1 (2004).

[2] A. Serebrov et al., Phys. Lett. B 605, 72 (2005).

[3] V. V. Nesvizhevsky, A. P. Serebrov, R. R. Taldaev, A. G. Kharitonov, V. P. Alfimenkov, A. V. Strelkov and V. N. Shvetsov, Sov. Phys. JETP 75, 405 (1992) [Zh. Eksp. Teor. Fiz. 102, 740 (1992)].

[4] H. Abele et al., Eur. Phys. J. C 33, 1 (2004).

[5] F. Mescia, arXiv:hep-ph/0411097.

[6] FJ Hartmann et al., proceedings of the Workshop on Quark-Mixing and CKMUnitarity, Heidelberg 2002, H. Abele and D. Mund eds., Mako-Verlag, Heidelberg 2003 and

R. Picker et al., J. Res. Natl. Inst. Stand. Technol., 110, \#4 (2005).

[7] R. Picker, Diplomarbeit TU-München, 2004, unpublished

[8] J. Bröcker, Diplomarbeit TU-München, 2004, unpublished 
[9] G. Dollinger et al., paper in preparation

[10] L. L. Nemenov, Sov. J. Nucl. Phys. 31, 1 (1980)

[11] B. G. Yerozolimsky, Nucl. Instr. and Methods in Physics Research A440, 491 (2000)

[12] N. Kaiser, TU-München, private communication February 2005, D. Dubbers, UniHeidelberg, private communication, February 2005.

[13] B. R. Holstein and S. B. Treiman, Phys. Rev. D 16, 2369 (1977).

[14] L. L. Nemenov, A. A. Ovchinnikova, Sov. J. Nucl. Phys. 31, 5 (1980)

[15] J. Byrne, Eur. Phys. Lett 56(5), 63 (2001)

[16] W. Haeberli, Ann. Rev. Nucl. Sci. 17, 373 (1967) and references quoted therein

[17] W. E. Lamb, R. C. Retherford, Phys. Rev. 79, 549 (1950)

[18] W. Gaubatz, TUM, private communication, 1998

[19] I. Altarev et al., internal report, TUM, 2000

[20] E. G. Adelberger et al., Phys. Rev. Lett. 83, 1299 (1999)

[21] H. P. Mumm, PhD thesis, Center for Experimental Nuclear Physics and Astrophysics, University of Washington, 2001 